\title{
An adaptive ERG technique to measure normal and altered dark adaptation in the mouse
}

\author{
Paul J. DeMarco Jr. • Yoshiaki Katagiri • \\ Volker Enzmann · Henry J. Kaplan • \\ Maureen A. McCall
}

Received: 20 March 2007 / Accepted: 6 August 2007/Published online: 22 September 2007

(C) Springer-Verlag 2007

\begin{abstract}
The time-course of dark adaptation provides valuable insights into the function and interactions between the rod and cone pathways in the retina. Here we describe a technique that uses the flash electroretinogram (ERG) response to probe the functional integrity of the cone and rod pathways during the dynamic process of dark adaptation in the mouse. Retinal sensitivity was estimated from the stimulus intensity required to maintain a $30 \mu \mathrm{V}$ criterion b-wave response during a $40 \mathrm{~min}$ period of dark adaptation. When tracked in this manner, dark adaptation functions in WT mice depended upon the bleaching effects of initial background adaptation conditions. Altered dark adaptation functions,
\end{abstract}

\section{P. J. DeMarco Jr. $(\bowtie)$}

Louisville VA Medical Center, Department of Psychological and Brain Sciences, University of Louisville, 317 Life Sciences Bldg., Louisville, KY, USA e-mail: paul.demarco@louisville.edu

Y. Katagiri · V. Enzmann · H. J. Kaplan

Department of Ophthalmology and Visual Sciences, University of Louisville, Louisville, KY, USA

Present Address:

V. Enzmann

Department of Ophthalmology, Inselspital, University of Bern, Bern 3010, Switzerland

\section{A. McCall}

Departments of Psychological and Brain Sciences and Ophthalmology and Visual Sciences, University of Louisville, Louisville, KY, USA commensurate with the functional deficit were recorded in pigmented mice that lacked cone function $\left(\right.$ Gnat $\left.^{\text {cplf3 }}\right)$ and in WT mice injected with a toxin, sodium iodate $\left(\mathrm{NaIO}_{3}\right)$, which targets the retinal pigment epithelium and also has downstream effects on photoreceptors. These data demonstrate that this adaptive tracking procedure measures retinal sensitivity and the contributions of the rod and/or cone pathways during dark adaptation in both WT control and mutant mice.

Keywords Animal model - Dark adaptation · Electroretinogram (ERG) · Mouse ·

Retina $\cdot$ Visual sensitivity

\section{Introduction}

The flash electroretinogram (ERG) is a widely used technique that evaluates retinal function and has become a standard tool for functional assessment of the retinal function [1, 2]. Its wide adoption stems from its straightforward technical implementation and many advances in the interpretation of its component waveforms [3-9]. It is amendable for use in a wide range of species, including humans, and as such bridges human clinical evaluations and animal models of retinal disease. Finally, the ERG technique is non-invasive, making it extremely useful for repeated measurements that evaluate changes in progressive disease forms. 
For a complete characterization, it is of value to assess both the rod and cone systems and thus, the ability of the retina to function under both dark (scotopic) and light adapted (photopic) conditions, respectively. These assessments are particularly important after experimental manipulation of elements in the rod or cone signaling pathways, or when screening animal models for mutations that alter normal function $[1,10]$. Like other mammals, the mouse retina contains both rod and cone photoreceptors [11], and as such, standardized protocols for independently recording the light and dark adapted mouse ERG have been proposed [1, 12, 13]. To isolate cone function, the retina is exposed to a background that desensitizes the rods, generating an ERG response dominated by cone initiated activity. Conversely, rod function is isolated by dark adaptation, which maximizes rod sensitivity, generating an ERG response dominated by rod initiated activity. In either case, ERG responses are recorded once the retina has achieved a steady state level of adaptation.

Alternative methods for recording the ERG from cone and rod pathways have been developed that measure retinal function under conditions in which the state of retinal adaptation is in flux. These techniques must utilize a metric that follows retinal sensitivity as a function of the changing adaptation state. For example, Peachey et al. [14] studied changes in cone-mediated sensitivity as a function of time after the onset of a photopic background were measured using the amplitude ERG b-wave. Similarly, the changing sensitivity of the retina after termination of a photopic background was examined in a line of VPP transgenic mice by measuring the growth of the ERG b-wave response [3]. The pairedflash technique [15] is another approach that has been used to study the recovery of the mouse rod response [16-18] as well as to assist in studying the mouse cone pathways in isolation $[19,20]$. In this paradigm, the ERG response is measured to a probe flash presented at varying intervals after a brighter (rod saturating) adapting flash. Each of these approaches uses the change in the ERG response amplitude to estimate retinal sensitivity. However, it is important to remember that the amplitude of the mouse ERG bwave is only linearly proportional to the stimulus intensity near threshold, while at higher stimulus intensities the response exhibits nonlinear saturation [9, 21]. Consequently, if ERG response amplitude is used to estimate retinal sensitivity, assumptions that link amplitude directly to changes in sensitivity are valid only when ERG responses are within the linear range.

Here, we describe an ERG recording technique that quantifies retinal sensitivity using a criterion response method. The advantage is that a low amplitude criterion response, applied through adaptation, ensures the linearity of the intensity response function and the link between ERG response amplitude as retinal sensitivity changes [22]. We applied this technique in the mouse to measure the process of dark adaptation and tracked the time-course of the shift from cone-dominated (photopic) vision to roddominated (scotopic) vision, including the transitional (mesopic) phase where both systems are operational [23]. As such, the measure provides an estimate of the relative sensitivity of the cone and rod systems in the mouse as the retina transitions from a photopic to a scotopic state. We describe use of this technique in wildtype (WT) mice and also show examples of its use to characterize adaptation in mice with known alterations in retinal function.

\section{Methods}

Animals

C57B1/6J (Jackson Laboratory, Bar Harbor, ME) served as WT controls and were between 60100 days of age at the time of recording. The ERG protocol was approved by the Institutional Animal Care and Use Committee at the University of Louisville and was performed in accordance with the Association for Research in Vision and Ophthalmology (ARVO) statement for the "Use of Animals in Ophthalmic and Vision Research". Experimental mice were age matched to the WT mice and were: (1) C57Bl/6J mice administered sodium iodate $\left(\mathrm{NaIO}_{3}\right)$ to damage the retinal pigment epithelium (RPE) and with it, rod and cone photoreceptor function, and (2) pigmented Gnat $2^{\text {cplf3 }}$ mice, which had been backcrossed onto a C57B1/6J background for five generations, and whose cone pathways had been inactivated due to a mutation [24].

The methods for $\mathrm{NaIO}_{3}$ administration and its effects on visual function have been described previously [25]. In brief, a sterile solution of $1 \%$ 
$\mathrm{NaIO}_{3}$ in saline was injected into the tail vein at a final dose of $35 \mathrm{mg} / \mathrm{kg}$. Adaptation was measured 2 weeks after injection.

\section{Electroretinography}

Mice were anesthetized with an intramuscular injection of a mixture of Ketamine $(27 \mathrm{mg} / \mathrm{kg})$ and Xylazine $(7 \mathrm{mg} / \mathrm{kg})$ dissolved in Ringer's solution. This dose maintained anesthesia for about 1 hour, the maximum duration for most adaptation experiments. For longer experiments, supplemental doses (80\% of the induction concentration) were administered at 50 min intervals after the initial dose. The pupil was dilated and accommodation relaxed with topical application of tropicamide (1\%) and phenylephrine hydrochloride $(2.5 \%)$. The mouse was mounted in a stereotaxic frame and a corneal contact lens was placed on the eye and used as the active electrode. To improve electrode contact, a layer of artificial tears (Polyvinyl Alcohol, 1.4\%) was applied to the cornea prior to placement of the contact lens. To avoid corneal drying the same artificial tear solution was delivered continuously $(45 \mu \mathrm{l} / \mathrm{h})$ during the experiment, using a syringe micropump (New Era Pump Systems, Inc.) connected to a blunt $25 \mathrm{Ga}$. needle placed just above the eye. Body temperature was maintained throughout the experiment at $37^{\circ} \mathrm{C}$ with a feedback regulated heating pad (Gayman, Inc.).

The ERG signal was recorded from the corneal surface with a contact lens that was embedded with a conductive silver thread electrode (DTL) [26]. A reference needle electrode was inserted in the forehead and a ground placed in the tail to complete the circuit. The flash ERG was recorded over a $500 \mathrm{~ms}$ epoch, amplified 10,000 times, bandpass filtered from 0.1 to $1,000 \mathrm{~Hz}$, and digitized at a sample rate of $3,000 \mathrm{~Hz}$.

Flash stimuli were produced using two $470 \mathrm{~nm}$ light emitting diodes (LED, Nichia Corp), whose lenses were reshaped so that they could be paired and directly imaged onto the end of a fiber-optic cable. The opposite end of the cable was positioned a few millimeters from the eye and formed a stimulus field of approximately $80^{\circ}$ of visual angle. Stimulus presentation and acquisition of the ERG responses were controlled by a program written in LabVIEW (National Instruments, Inc.), which interfaced with data acquisition hardware. The program controlled the stimulus duration and intensity through an interface with custom-built electronic drivers. Each LED light output was controlled independently and was a linear function of drive voltage over a $10 \mathrm{~V}$ range. Stimulus output was calibrated on a regular basis in radiometric units, and in both scotopic and photopic photometric units with a radiometer (IL 1700, International Light, Inc.).

At each stimulus intensity the ERG was evoked by a continuous train of $10 \mathrm{~ms}$ flashes, presented at $2 \mathrm{~Hz}$, producing a recording epoch of $500 \mathrm{~ms}$ for each ERG response. To enhance the accuracy of the sensitivity measurements, we averaged 10 ERG responses over each subsequent train of 10 epochs, yielding real-time estimates of retinal sensitivity at $5 \mathrm{~s}$ intervals over the $40 \mathrm{~min}$ tracking period. Amplitude was measured from the trough of the awave (or baseline in the absence of an a-wave) to the peak of $b$-wave. The software program measured ERG amplitude and altered stimulus intensity to maintain a criterion ERG response of $30 \mu \mathrm{V}$. Under our conditions, the b-wave peak occurred about $50 \mathrm{~ms}$ after the flash. Using this information, the software was programmed to search for the b-wave peak within $30-80 \mathrm{~ms}$ of the stimulus presentation, which avoided artifacts in these measurements. If the ERG response was not within $10 \%$ of the $30 \mu \mathrm{V}$ criterion, the program altered the stimulus intensity for next set of trials, increasing or decreasing stimulus intensity in $0.05 \mathrm{log}$ unit steps depending on whether the amplitude was larger or smaller than criterion. Thus, the adaptive tracking procedure produces a stimulus that evokes a constant b-wave response amplitude throughout a period of $40 \mathrm{~min}$, a time frame in which dark adaptation measured using the ERG is reported to stabilize [3].

WT controls were exposed to one of two adapting backgrounds $\left(2.4 \log \mathrm{cd} \mathrm{m}^{-2}\right.$ or $4.0 \log \mathrm{cd} \mathrm{m}^{-2}$ ) for $10 \mathrm{~min}$. The adapting field was created with a $75 \mathrm{~W}$ xenon arc lamp source and imaged on the retina via a separate fiber optic cable subtending the same visual angle as the test stimuli. At the end of the adaptation period, the background was extinguished and replaced with a separate fiber optic that imaged the LED stimulus and the tracking procedure was initiated. At the start of the tracking program both LEDs flashed in tandem at a combined flash intensity of 17.5 scotopic (scot) $\mathrm{cd} \mathrm{s} \mathrm{m}^{-2}$, to generate the 
criterion response. As retinal sensitivity increased with time in the dark, the flash intensity for one LED was decreased while the other continued at peak output. Near the end of the adaptation period only a single LED was required to maintain the criterion response. When mice initially dark adapted, the initial intensity of the tracking stimulus was lowered to accommodate increased retinal sensitivity by placing a neutral density filter between the LEDs and the fiber optic cable.

Statistical analyses

Group differences were analyzed with a two-tailed Student's test for independent groups. To compare the ultimate sensitivity in each condition, the stimulus intensity that elicited the criterion response over the last 2 min of the dark adaptation function was averaged for each mouse and used to compute the group average.

\section{Results}

Dark adaptation in WT mice

Figure 1a plots individual adaptation functions for nine WT mice (colored lines) and their average (black line) for the $2.4 \log \mathrm{cd} \mathrm{m}^{-2}$ adapting condition. In each, flash intensity decreases with increasing time in the dark to maintain a $30 \mu \mathrm{V}$ criterion response. Retinal sensitivity increases rapidly over the first $5 \mathrm{~min}$; thereafter, the slope of the curve declines but retinal sensitivity continues to increase and becomes stable by $40 \mathrm{~min}$. It is well know that recovery of visual sensitivity after a bleaching stimulus is inversely related to the product of the light intensity and stimulus duration [27]. From previously published calculations of the effectiveness of light on rhodopsin bleaching in vivo for the WT mouse eye [28], we estimate that a $2.4 \log \mathrm{cd} \mathrm{m}^{-2}$ background bleaches approximately $50 \%$ of the available rhodopsin in the retina.

When a $4.0 \log \mathrm{cd} \mathrm{m}^{-2}$ initial background was used that bleaches over $95 \%$ of the available rhodopsin, the resulting adaptation profile was quite different (Fig. 1b;n=8). Both the shape of the adaptation function and the sensitivity of the retina at
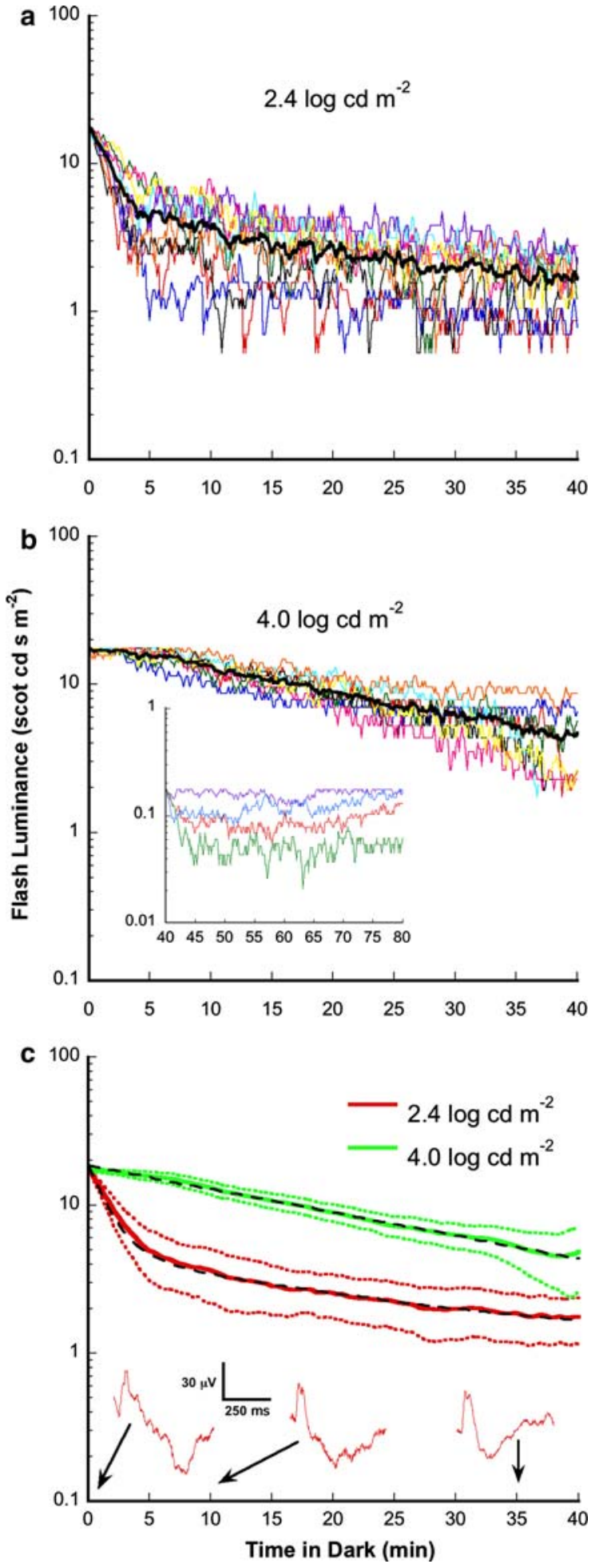
4 Fig. 1 Dark adaptation differs as a function of the adapting background in WT mice. Adaptation functions for WT mice exposed to a background of either: (a) $2.4 \log \mathrm{cd} \mathrm{m}^{-2}(n=9)$ or (b) $4.0 \log \mathrm{cd} \mathrm{m}^{-2}(n=8)$ for $10 \mathrm{~min}$ prior to the start of the tracking program. Data for individual mice (colored lines) and the mean for each group (black line) are shown for each condition. These backgrounds are estimated to bleach approximately $50 \%$ and $100 \%$ of rhodopsin in the mouse eye, respectively. The inset in Fig. $1 \mathrm{~b}$ plots dark adaptation functions, using the tracking program, for mice $(n=4)$ adapted to a $4.0 \log \mathrm{cd} \mathrm{m}^{-2}$ background for $10 \mathrm{~min}$ and then dark adapted for $40 \mathrm{~min}$ (see text for details). (c) The mean curves for each background condition, along with $95 \%$ confidence intervals show the differences in adaptation and final sensitivity reach after exposure to the two backgrounds. The dotted lines represent exponential decay functions fit to each group mean (see text for details). Representative averaged ERG waveforms from one mouse in the $2.4 \log \mathrm{cd} \mathrm{m}^{-2}$ background condition at 1,10 and $35 \mathrm{~min}$ after termination of the background. Each waveform is $500 \mathrm{~ms}$ in duration and trough to peak b-wave amplitude is approximately $30 \mu \mathrm{V}$

40 min differ when the curves from the two adapting backgrounds are compared. Figure 1c compares the mean curves for the two adapting conditions, smoothed by a Hanning method, along with their with $95 \%$ confidence intervals. The mean dark adaptation curves for each background condition were fit by exponential decay functions, which are indicated by the black dotted lines. The dark adaptation function for the $2.4 \log \mathrm{cd} \mathrm{m}^{-2}$ adapting background is best modeled by a two process equation that captures both an initial fast phase and a secondary slower phase, with time constants of $4.5 \mathrm{~min}$ and $57.4 \mathrm{~min}$, respectively. In contrast, the dark adaptation function for the $4.0 \mathrm{log} \mathrm{cd} \mathrm{m}^{-2}$ adapting background could be modeled by a single process equation whose time constant was $74.9 \mathrm{~min}$, suggesting that the initial fast process seen for the dimmer adapting background is saturated by the brighter background. Thus, these differences show that the technique can be used to track dark adaptation under various bleaching conditions and processes with different time-courses. The insets in Fig. 1c are examples of averaged ERG waveforms recorded at 1, 10 and $35 \mathrm{~min}$ for the $2.4 \mathrm{log} \mathrm{cd} \mathrm{m}^{-2}$ adapting background. Early in dark adaptation, the ERG waveforms had prominent a- and b-wave components, typical of a mixed rod-cone (mesopic) response in mouse [3, 20]. Waveforms sampled from later time points had b-wave but small or absent awaves, typical of a rod-dominated response to lower intensity stimuli $[3,8,29]$.
As a control, a separate group of four WT mice were exposed to the $4.0 \mathrm{log} \mathrm{cd} \mathrm{m}^{-2}$ adapting background for $10 \mathrm{~min}$ and, then placed in total darkness for $40 \mathrm{~min}$. At the end of the dark adaptation period, the tracking program was started (with no additional adaptation), and sensitivity was tracked for an additional $40 \mathrm{~min}$. We used this to evaluate the degree to which the tracking protocol, in itself, influenced the time-course of dark adaptation. The data, shown in the inset in Fig. 1b, demonstrate that the sensitivity reached after 40 min of dark adaptation is stable albeit higher than during the $40 \mathrm{~min}$ tracking period (Fig. 1b). The mean flash luminance required to elicit a criterion ERG response at $40 \mathrm{~min}$ in Fig. $1 \mathrm{~b}$, is $1.7 \mathrm{scot} \mathrm{cd} \mathrm{s} \mathrm{m}^{-2}$, compared to $0.1 \mathrm{scot}$ cd s m${ }^{-2}$ after 40 min of dark adaptation (inset), a significant reduction in sensitivity of $1.2 \mathrm{log}$ units $(t=2.6, P<.05)$.

Mice with dysfunctional cones have delayed dark adaptation

If our tracking program assesses first cone and then rod function, then the overall shape of the curve should be altered in the absence of one photoreceptor type. To test our technique and this hypothesis, we assessed dark adaptation in mice with a mutation in the Gnat $2^{\text {cplf3 }}$ gene, which have abnormal cone function as early as 4 weeks of age due to an interruption in the phototransduction cascade [24], but assessments of rod function appear to be within the normal range [24, 30, 31]. When backgrounds greater than 1.5 and, as high as $2.0 \log \mathrm{cd} \mathrm{m}^{-2}$, were used, we found that the ERG response of Gnat $2^{\text {cplf3 }}$ mice remained saturated for over $40 \mathrm{~min}$. Therefore, we adapted mice for $5 \mathrm{~min}$ to a $1.2 \mathrm{~cd} \mathrm{~m}^{-2}$ background because with this combination the ERG typically regained sensitivity within our standard 40 tracking period. Figure 2 shows dark adaptation for four Gnat $2^{\text {cplf3 }}$ mice at 2 months of age (colored lines), along with the mean for three WT mice (black line) recorded under the same adapting conditions. The Gnat $2^{\text {cplf3 }}$ mice show delayed dark adaptation; the ERG response from these mice remains saturated for up to $20 \mathrm{~min}$, but then begins to regain sensitivity, reaching approximately the same sensitivity of WT mice at the end of $40 \mathrm{~min}$. The change in the $\mathrm{x}$-axis scale in Fig. 2, relative to other plots in this study, 


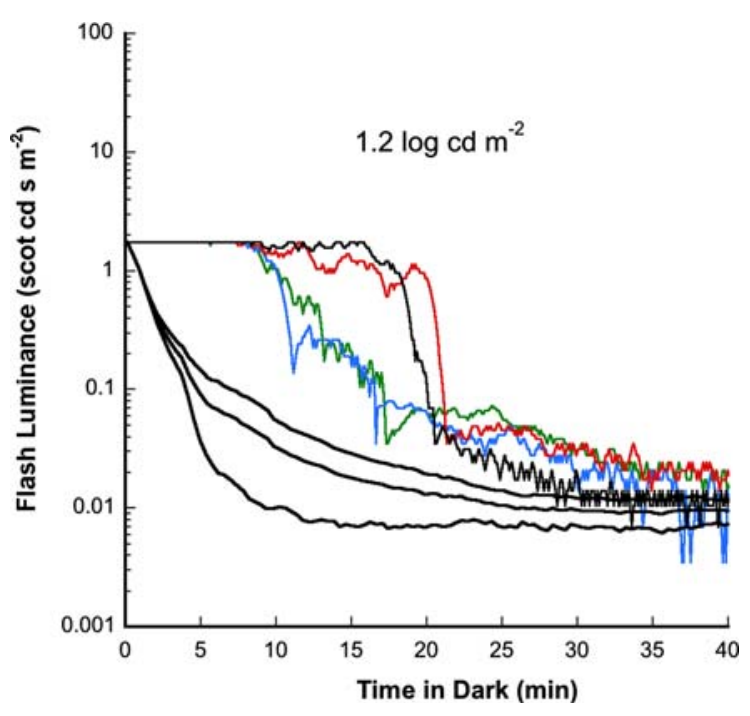

Fig. 2 Dark adaptation is altered by a spontaneous mutation. Dark adaptation functions measured in a pigmented strain of mice that have a mutation in the Gnat 2 gene, causing a functional loss of cone responses. Before testing, mice were adapted to a background of $1.3 \mathrm{log} \mathrm{cd} \mathrm{m}^{-2}$ for $5 \mathrm{~min}$. Functions from four mutant mice are shown in color, while the average and $95 \%$ confidence intervals of three WT mice are shown in black. Gnat $2^{\text {cplf3 }}$ mice have delayed dark adaptation, owning to the loss of cone function and sustained saturation of rod response, for up to $20 \mathrm{~min}$, before beginning the dark adaptation process

reflects the lower starting intensity for the tracking stimulus needed to promote rod recovery. In addition, WT mice using these conditions reach a higher stable sensitivity after 40 min compared to the two brighter backgrounds we tested (Fig. 1c).

$\mathrm{NaIO}_{3}$ model of RPE damage produces changes in both the time-course and overall sensitivity of dark adaptation

We have previously studied the effects of $\mathrm{NaIO}_{3}$ treatment on visual function in mice [25]. $\mathrm{NaIO}_{3}$ damages the RPE and, as a consequence, alters both rod and cone function [32-34]. As an extension of our previous work, we evaluated adaptation in $\mathrm{NaIO}_{3}$-treated mice using a $2.4 \log \mathrm{cd} \mathrm{m}^{-2}$ adapting background. Figure 3 plots the changes in retinal sensitivity in three $\mathrm{NaIO}_{3}$ treated mice, along with the average and $95 \%$ confidence intervals from our WT data for comparison. $\mathrm{NaIO}_{3}$ treated mice show a significant decrease in sensitivity $(t=3.3, P<.01)$

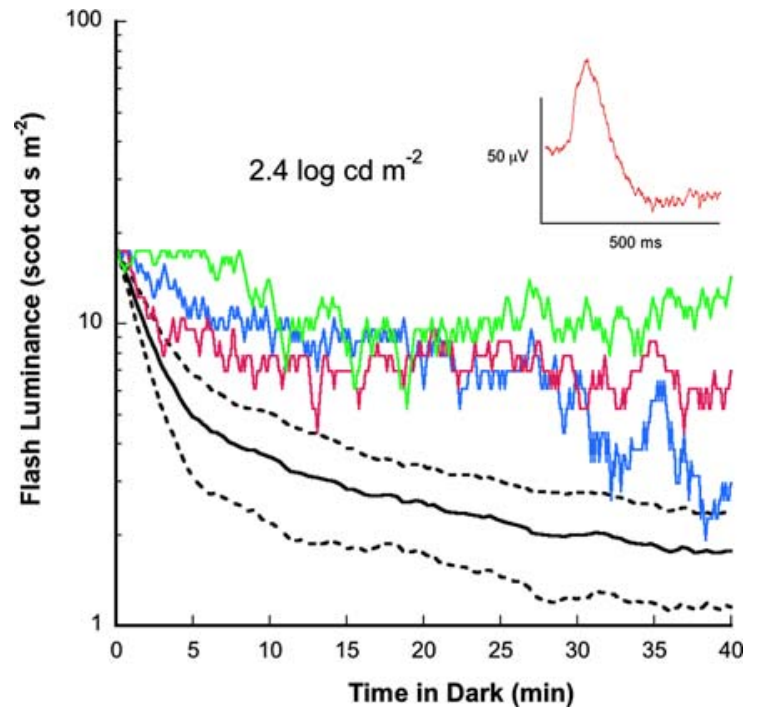

Fig. 3 Dark adaptation is altered by a chemical treatment which is toxic to the retinal pigment epithelium. Dark adaptation functions for mice after adapting to the $2.4 \mathrm{log}$ cd $\mathrm{m}^{-2}$ background for mice (colored lines; $n=3$ ) treated with $\mathrm{NaIO}_{3}(35 \mathrm{mg} / \mathrm{kg}) 2$ weeks prior to testing. Black lines show the WT mean and $95 \%$ confidence intervals under the same conditions. At the end of tracking, the mean sensitivity of $\mathrm{NaIO}_{3}$ treated mice is $0.5 \mathrm{log}$ units lower than WT. The inset shows a representative flash ERG from a $\mathrm{NaIO}_{3}$ treated mouse

reflecting the functional deficits that we have previously observed, using other measures of retina structure and visual function [25]. A typical flash ERG waveform from a $\mathrm{NaIO}_{3}$ treated mouse (inset of Fig. 3) illustrates a normally shaped ERG (although it is significantly smaller than control).

\section{Discussion}

We describe the use of a non-invasive ERG method using a low-amplitude fixed response criterion to assess changing sensitivity of the retina as a function of dark adaptation in the mouse retina. Using this technique, we compare dark adaptation in WT mice with those lacking functional cones and in mice with diminished photoreceptor function to show the relative contribution of cones and rods during the transition from photopic to scotopic vision.

The dark adaptation function for WT mice after adaptation to a $2.4 \log \mathrm{cd} \mathrm{m}^{-2}$ background is best fit by a two-process exponential decay function (Fig. 1a). The initial limb has a fast decay, while the second is 
significantly slower. A similarly shaped function is obtained for WT mice when a $1.3 \log \mathrm{cd} \mathrm{m}^{-2}$ background is used (Fig. 2-black trace). The data from our WT mice and from our comparisons of dark adaptation across WT, Gnat $2^{\text {cplf3 }}$ mutant and $\mathrm{NaIO}_{3}$ treated mice provide strong evidence that cone function contributes to the first limb of the WT function, while the second limb and final sensitivity rely on rod function. First, the initial insensitivity in nnat $^{\text {cplf3 } 3}$ mice suggests that in the absence of cone function the rod system is saturated and requires time to recover (Fig. 2). The final sensitivity in Gnat $2^{\text {cplf3 }}$ mutants is similar to WT, indicating a contribution of the rod system. Second, the light intensity (1.7 $\mathrm{scot} \mathrm{cd} \mathrm{s} \mathrm{m}^{-2}$ ) required for a criterion response in the WT retina at $40 \mathrm{~min}$ (Fig. 1a) is within the range reported previously to elicit a rod-dominated ERG b-wave response in the dark adapted mouse [9] and further indicates that the second limb can be attributed to rod function. Third, the adaptation functions from Gnat $^{\text {cplf3 }}$ and $\mathrm{NaIO}_{3}$ mice differ. The $\mathrm{NaIO}_{3}$-treated retina is generally less sensitivity throughout testing, which is consistent with dysfunction and loss of both rod and cone photoreceptors due RPE destruction. A similar general effect on sensitivity also was observed in the Royal College of Surgeons rat, in which the RPE is dysfunctional [22]. Finally, when photoreceptors are bleached with a bright adapting background (4.0 log cd $\mathrm{m}^{-2}$ ), the resulting adaptation function is fit by a single exponential decay function. This is consistent with observations across a number of species, that the timecourse of the recovery of ERG sensitivity is related to extent of bleaching of the photopigment (mouse [16, $35,36]$, rat [22] and human [37]).

The dark adaptation function of the WT mouse is best fit by a two component exponential decay function. However, the limbs of the curve in the mouse and rat [22] are less distinct than in the human [38-40]. A contributing factor to this species difference arises from the similarity of the peak wavelengths in the rod and M-cone pigments in the rodent, a difference of only about $7 \mathrm{~nm}$ [41]. In the human the difference between rod and L-cone pigment peak sensitivities can be over $50 \mathrm{~nm}$ [4244]. This large difference can exploited to enhance the prominence of the cone-rod break by choosing a long-wavelength test stimulus that is detected more easily by L-cones initially, and later by rods as their sensitivity surpasses the cones during dark adaptation. An analogous method cannot be used in mice because of the spectral similarity between rod and M-cone pigments, and the overlap of UV-cone and rod pigments at shorter wavelengths.

The advantage of the adaptive technique we describe is that it relies upon maintaining a criterion response amplitude as dark adaptation progresses and directly measures the increase in retinal sensitivity as a function of time. A very similar method to the one developed here has been used with humans to measure dark adaptation of the cone systems [45]. An alternative ERG method has been described that assesses dark adaptation in the mouse uses a constant intensity stimulus [3, 16]. Both methods estimate sensitivity by measuring b-wave amplitude, but the adaptive technique keeps amplitude constant but the other estimates the increase in sensitivity of the rod system indirectly from the growth of the b-wave amplitude. Both techniques also require initial empirical work to determine starting stimulus parameters. In our case, the level of adaptation must be selected so that it does not saturate the system, while the latter must determine the proper starting stimulus intensity, so that it is both bright enough to evoke a response from the light adapted retina, but not so bright as to cause response saturation before dark adaptation is complete. In the latter method, difficulty may arise in linking the ERG response to retinal sensitivity when the non-linear response range is reached, near b-wave response saturation when a fixed stimulus intensity is used. In contrast, the use of a criterion, assures that the response remains within a linear response range.

While our adaptive technique has advantages, it also is limited in its ability to estimate absolute sensitivity. In the mouse, the absolute threshold for a criterion b-wave response has been reported require a stimulus flash of about $-5 \log \operatorname{scot} t d s$ [8]. Our lowest mean dark adapted threshold is obtained with a flash of $0.01(-2 \mathrm{log}) \mathrm{scot} \mathrm{cd} \mathrm{s} \mathrm{m}^{-2}$ stimulus (Fig. 2), which is $3 \log$ units higher, indicating that our stimuli maintain the retina at levels higher than absolute threshold. We also show that the tracking stimulus does not further desensitize the retina after a prolonged period of dark adaptation (Fig. 1b, inset). However, comparing retinal sensitivity after $40 \mathrm{~min}$ of the tracking procedure (Fig. 1b) to 40 min of dark adaptation without the tracking procedure (Fig. 1b inset) suggests that the tracking procedure may cause some delay in the normal time-course of adaptation. 
Modifications could be made to increase ultimate sensitivity, e.g., lower initial intensities for the adapting background and tracking stimulus or increasing inter-stimulus interval. However, each will, in some way, compromise the contribution of the cone system in the analysis.

We have shown that an automated adaptive technique, which measures the ERG recorded from the mouse eye, is a valuable tool for determining the properties of dark adaptation in WT mice and those with alterations in visual function. Judicious choice of initial stimulus parameters should allow this technique to transfer to other studies of the rod and cone pathway contribution in other mouse mutants and in species other than rodents.

Acknowledgements Supported by: The Department of Veterans Affairs (DeMarco) and NIH EY014701 (McCall). We would like to thank Jennifer Brodfuerer, Donald Ladwig and Alex Thomas for assistance in data collection. We also thank Dr. Bo Chang and Norman Hawes for providing Gnat $2^{\text {cplf3 }}$ mice.

\section{References}

1. Peachey NS, Ball SL (2003) Electrophysiological analysis of visual function in mutant mice. Doc Ophthalmol 107:13-36

2. Pinto LH, Enroth-Cugell C (2000) Tests of the mouse visual system. Mamm Genome 11:531-536

3. Goto Y, Peachey NS, Ripps H, Naash MI (1995) Functional abnormalities in transgenic mice expressing a mutant rhodopsin gene. Invest Ophthalmol Vis Sci 36:62-71

4. Friedburg C, Allen CP, Mason PJ, Lamb TD (2004) Contribution of cone photoreceptors and post-receptoral mechanisms to the human photopic electroretinogram. J Physiol 556:819-834

5. Frishman LJ, Sieving PA (1995) Evidence for two sites of adaptation affecting the dark-adapted ERG of cats and primates. Vision Res 35:435-442

6. Niemeyer G (2005) ERG components of negative polarity from the inner retina and the optic nerve response. Doc Ophthalmol 111:179-189

7. Rangaswamy NV, Frishman LJ, Dorotheo EU, Schiffman JS, Bahrani HM, Tang RA (2004) Photopic ERGs in patients with optic neuropathies: comparison with primate ERGs after pharmacologic blockade of inner retina. Invest Ophthalmol Vis Sci 45:3827-3837

8. Saszik SM, Robson JG, Frishman LJ (2002) The scotopic threshold response of the dark-adapted electroretinogram of the mouse. J Physiol 543:899-916

9. Robson JG, Maeda H, Saszik SM, Frishman LJ (2004) In vivo studies of signaling in rod pathways of the mouse using the electroretinogram. Vision Res 44:3253-3268
10. Chang B, Hawes NL, Hurd RE, Wang J, Howell D, Davisson MT, Roderick TH, Nusinowitz S, Heckenlively JR (2005) Mouse models of ocular diseases. Vis Neurosci 22:587-593

11. Carter-Dawson LD, LaVail MM (1979) Rods and cones in the mouse retina. I. Structural analysis using light and electron microscopy. J Comp Neurol 188:245-262

12. Bayer AU, Cook P, Brodie SE, Maag KP, Mittag T (2001) Evaluation of different recording parameters to establish a standard for flash electroretinography in rodents. Vision Res 41:2173-2185

13. Rosolen SG, Rigaudiere F, Le Gargasson JF, Brigell MG (2005) Recommendations for a toxicological screening ERG procedure in laboratory animals. Doc Ophthalmol 110:57-66

14. Peachey NS, Goto Y, al-Ubaidi MR, Naash MI (1993) Properties of the mouse cone-mediated electroretinogram during light adaptation. Neurosci Lett 162:9-11

15. Birch DG, Hood DC, Nusinowitz S, Pepperberg DR (1995) Abnormal activation and inactivation mechanisms of rod transduction in patients with autosomal dominant retinitis pigmentosa and the pro-23-his mutation. Invest Ophthalmol Vis Sci 36:1603-1614

16. Lyubarsky AL, Pugh EN Jr. (1996) Recovery phase of the murine rod photoresponse reconstructed from electroretinographic recordings. J Neurosci 16:563-571

17. Goto Y, Peachey NS, Ziroli NE, Seiple WH, Gryczan C, Pepperberg DR, Naash MI (1996) Rod phototransduction in transgenic mice expressing a mutant opsin gene. J Opt Soc Am A Opt Image Sci Vis 13:577-585

18. Ueda Y, Tammitsu N, Imai H, Honda Y, Shichida Y (2006) Recovery of rod-mediated a-wave during lightadaptation in mGluR6-deficient mice. Vision Res 46:16551664

19. Lyubarsky AL, Falsini B, Pennesi ME, Valentini P, Pugh EN Jr. (1999) UV- and midwave-sensitive cone-driven retinal responses of the mouse: a possible phenotype for coexpression of cone photopigments. J Neurosci 19:442455

20. Lyubarsky AL, Chen C, Simon MI, Pugh EN Jr. (2000) Mice lacking G-protein receptor kinase 1 have profoundly slowed recovery of cone-driven retinal responses. J Neurosci 20:2209-2217

21. Fulton AB, Manning KA, Baker BN, Schukar SE, Bailey CJ (1982) Dark-adapted sensitivity, rhodopsin content, and background adaptation in pcd/pcd mice. Invest Ophthalmol Vis Sci 22:386-393

22. Perlman I (1978) Dark-adaptation in abnormal (RCS) rats studied electroretinographically. J Physiol 278:161-175

23. Stockman A, Sharpe LT (2006) Into the twilight zone: the complexities of mesopic vision and luminous efficiency. Ophthalmic Physiol Opt 26:225-239

24. Chang B, Dacey MS, Hawes NL, Hitchcock PF, Milam AH, Atmaca-Sonmez P, Nusinowitz S, Heckenlively JR (2006) Cone photoreceptor function loss-3, a novel mouse model of achromatopsia due to a mutation in Gnat2. Invest Ophthalmol Vis Sci 47:5017-5021

25. Enzmann V, Row BW, Yamauchi Y, Kheirandish L, Gozal D, Kaplan HJ, McCall MA (2006) Behavioral and anatomical abnormalities in a sodium iodate-induced 
model of retinal pigment epithelium degeneration. Exp Eye Res 82:441-448

26. Sagdullaev BT, DeMarco PJ, McCall MA (2004) Improved contact lens electrode for corneal ERG recordings in mice. Doc Ophthalmol 108:181-184

27. Lamb TD, Pugh EN Jr. (2004) Dark adaptation and the retinoid cycle of vision. Prog Retin Eye Res 23:307-380

28. Lyubarsky AL, Daniele LL, Pugh EN Jr. (2004) From candelas to photoisomerizations in the mouse eye by rhodopsin bleaching in situ and the light-rearing dependence of the major components of the mouse ERG. Vision Res 44:3235-3251

29. Smith SB, Hamasaki DI (1994) Electroretinographic study of the C57BL/6-mivit/mivit mouse model of retinal degeneration. Invest Ophthalmol Vis Sci 35:3119-3123

30. Nathan J, Reh R, Ankoudinova I, Ankoudinova G, Chang B, Heckenlively J, Hurley JB (2006) Scotopic and photopic visual thresholds and spatial and temporal discrimination evaluated by behavior of mice in a water maze. Photochem Photobiol 82:1489-1494

31. Nusinowitz S, Ridder WH 3rd, Ramirez J (2007) Temporal response properties of the primary and secondary rod-signaling pathways in normal and Gnat2 mutant mice. Exp Eye Res 84:1104-1114

32. Adachi-Usami E, Mizota A, Ikeda H, Hanawa T, Kimura T (1992) Transient increase of b-wave in the mouse retina after sodium iodate injection. Invest Ophthalmol Vis Sci 33:3109-3113

33. Mizota A, Adachi-Usami E (1997) Functional recovery of retina after sodium iodate injection in mice. Vision Res 37:1859-1865

34. Kiuchi K, Yoshizawa K, Shikata N, Moriguchi K, Tsubura A (2002) Morphologic characteristics of retinal degeneration induced by sodium iodate in mice. Curr Eye Res 25:373-379
35. Hetling JR, Pepperberg DR (1999) Sensitivity and kinetics of mouse rod flash responses determined in vivo from paired-flash electroretinograms. J Physiol 516(Pt 2):593609

36. Kang Derwent JJ, Qtaishat NM, Pepperberg DR (2002) Excitation and desensitization of mouse rod photoreceptors in vivo following bright adapting light. J Physiol 541:201218

37. Cameron AM, Mahroo OA, Lamb TD (2006) Dark adaptation of human rod bipolar cells measured from the b-wave of the scotopic electroretinogram. J Physiol 575:507-526

38. Haig C (1941) The course of rod dark adaptation as influenced by the intensity and duration of pre-adaptation to light. J Gen Physiol 24:735-751

39. Hecht S, Haig C, Chase AM (1937) The influence of light adaptation on subsequent dark adaptation of the eye. J Gen Physiol 20:831-850

40. Pugh EN (1975) Rushton's paradox: rod dark adaptation after flash photolysis. J Physiol 248:413-431

41. Jacobs GH, Williams GA, Fenwick JA (2004) Influence of cone pigment coexpression on spectral sensitivity and color vision in the mouse. Vision Res 44:1615-1622

42. Kraft TW, Schneeweis DM, Schnapf JL (1993) Visual transduction in human rod photoreceptors. J Physiol 464:747-765

43. Schnapf JL, Kraft TW, Baylor DA (1987) Spectral sensitivity of human cone photoreceptors. Nature 325:439-441

44. DeMarco P, Pokorny J, Smith VC (1992) Full-spectrum cone sensitivity functions for X-chromosome-linked anomalous trichromats. J Opt Soc Am A 9:1465-1476

45. Norren DV, Padmos P (1974) Dark adaptation of separate cone systems studied with psychophysics and electroretinography. Vision Res 14:677-686 